FIAN/TD-22/11

ITEP/TH-01/12

\title{
Interplay between MacDonald and Hall-Littlewood expansions of extended torus superpolynomials
}

\author{
A.Mironov ${ }^{*}$ A.Morozov ${ }^{\dagger}$ Sh.Shakirov ${ }^{\ddagger}$ and A.Sleptsov ${ }^{\S}$
}

\begin{abstract}
In [1] extended superpolynomials were introduced for the torus links $T[m, m k+r]$, which are functions on the entire space of time variables and, at expense of reducing the topological invariance, possess additional algebraic properties, resembling those of the matrix model partition functions and the KP/Toda tau-functions. Not surprisingly, being a suitable extension it actually allows one to calculate the superpolynomials. These functions are defined as expansions into MacDonald polynomials, and their dependence on $k$ is entirely captured by the action of the cut-and-join operator, like in the HOMFLY case. We suggest a simple description of the coefficients in these character expansions, by expanding the initial (at $k=0)$ conditions for the $k$-evolution into the new auxiliary basis, this time provided by the Hall-Littlewood polynomials, which, hence, play a role in the description of the dual $m$-evolution. For illustration we list manifest expressions for a few first series, $m k \pm 1, m k \pm 2, m k \pm 3, m k \pm 4$. Actually all formulas were explicitly tested up to $m=17$ strands in the braid.
\end{abstract}

\section{Introduction}

In [1] a general expression was suggested for the superpolynomials $[2,3]$ of the torus knots and links, which is actually a W-representation of [4] (ultimately related with matrix model representations [5] and Hurwitz theory [4]), generalizing the known expression of this kind $[6,7]$ for the torus HOMFLY polynomials, of which it is actually a $\beta$-deformation [8] with $t=q^{\beta}$. It directly reproduces all available answers for particular torus knots obtained by several alternative methods in $[9,10,11,12,13]$. It is naturally generalized to the colored superpolynomials, but, in this letter, we restrict consideration to the case of the first fundamental representation $R=\square=[1]$.

The main idea is to extend superpolynomials to functions of a $\tau$-function type, depending on infinitely many time variables $p_{k}$, which are no longer knot invariants (they depend on the braid representation of the knot), but instead is a nice algebraic quantity, possessing a variety of hidden symmetries. It has natural form of a character decomposition, which, in the $\beta$-deformed case, is the decomposition into the MacDonald polynomials $M_{Q}\{p\}$,

$$
\mathcal{P}_{m, m k+r}\{p\} \quad=\quad \sum_{Q \vdash m} c_{Q}^{(m, r)} q^{-k \nu\left(Q^{T}\right)} t^{k \nu(Q)} M_{Q}\{p\}=e^{k \hat{W}} \mathcal{P}_{m, r}\{p\}
$$

where $[m, n]=[m, m k+r], \quad 0 \leq r<m$ dentes the torus link, $Q=\left\{Q_{1} \geq Q_{2} \geq \ldots \geq Q_{l(Q)}>0\right\}$ are the Young diagrams of the size $\sum_{i=1}^{l(Q)} Q_{i}=m$ with $l(Q)$ rows, $Q^{T}$ is the transposed diagram of $Q$, and $\nu(Q)=\sum_{i=1}^{l(Q)}(i-1) Q_{i}$. Both $M_{Q}$ and $c_{Q}$ also depend on the two deformation parameters $q$ and $t=q^{\beta}$.

* Lebedev Physics Institute and ITEP, Moscow, Russia; mironov@itep.ru; mironov@lpi.ru

$\dagger$ ITEP, Moscow, Russia; morozov@itep.ru

$¥$ Department of Mathematics, University of California, Berkeley, USA, Center for Theoretical Physics, University of California, Berkeley, USA and ITEP Moscow, Russia; shakirov@math.berkeley.edu

§ITEP, Moscow, Russia; sleptsov@itep.ru 
This $\mathcal{P}_{m, n}\{p\}$ reduces to an ordinary superpolynomial $P_{m, n}(A)=\mathcal{P}_{m, n}\left\{p=p^{*}\right\}=\mathcal{P}_{m, n}^{*}$ at a special $1 d$ locus in the space of time variables, parameterized by $A=t^{N}$,

$$
p_{k}=p_{k}^{*}=\frac{1-A^{k}}{1-t^{k}}=[N]_{t^{k}}
$$

It becomes a polynomial with all coefficients positive in the case of knot (for $m$ and $n$ coprime) and after being expressed in the special variables ${ }^{1}$

$$
\mathbf{t}=-\sqrt{q / t}, \quad \mathbf{q}=\sqrt{t}, \quad \mathbf{a}^{2}=A \sqrt{t / q}
$$

A non-trivial part of the story is a description of the expansion coefficients $c_{Q}$. For the HOMFLY polynomials these were essentially $q$-independent integers, read off from the Adams decomposition at the "initial" point $n=0$ [6]. After $\beta$-deformation they become non-trivial rational functions of $q$ and $t$, and are not given by just a naive deformation of the Adams rule [1]. As explicitly stated in (1) they do not depend on the "evolution" parameter $k$, only on the "series" labeled by the residue $r=n \bmod m$. These coefficients are straightforwardly calculated, by using

- the duality $\mathcal{P}_{m, n}^{*}(A) \sim A^{m-n} \mathcal{P}_{n, m}^{*}(A)$ at the "initial" point $k=0$, i.e. $n=r$ : it provides a recurrent relation in $m$, allowing one to go down from $m$ to $m^{\prime}=r<m$, and

- the lifting rules, allowing to continue the superpolynomial at the "initial" point $k=0$ from the locus $\left\{p^{*}\right\}$ to the entire $\{p\}$-space;

- another initial condition at $k=-1$ can be used to additionally test the results; to do so one should use the symmetries $T[m,-n] \leftrightarrow T[m, n]$ and $T[m, r] \leftrightarrow T[m, m-r]$, see formula (23).

All this is explained and illustrated in great detail in [1], and an important problem is to find a convenient description of the rather sophisticated combinatorial functions $c_{Q}^{(m, r)}$. In this letter, we suggest a possibility which looks very promising. The key observation is that the extended superpolynomial (1) at $k=0$ has a nice decomposition in terms of the Hall-Littlewood polynomials $L_{Q}\{p\}=\left.M_{Q}\{p\}\right|_{q=0}$ :

$$
\mathcal{P}_{m, r}\{p\}=\sum_{Q \vdash m} c_{Q}^{(m, r)} M_{Q}\{p\}=\sum_{\substack{Q \vdash m \\ l(Q) \leq r}} h_{Q}^{(m, r)} L_{Q}\{p\}
$$

namely the coefficients $h_{Q}$ are non-vanishing only for $l(Q) \leq r$ (no more than $r$ rows) and possess additional algebraic properties. We list now the generating function for these coefficients for a few lowest values of $r$ to illustrate this statement.

\section{Hall-Littlewood coefficients $h_{Q}$ for torus knots}

The coefficients $h_{Q}$ are in many ways simpler than the coefficients $c_{Q}$. In particular, in variance with $c_{Q}$, the coefficients $h_{Q}$ are polynomials in $q, t$ with integer coefficients:

$$
h_{Q} \in \mathbb{Z}[q, t]
$$

It is convenient to separate a simple overall factor

$$
h_{Q}=q^{\nu(Q)}(1-t)^{l(Q)-1} \hat{h}_{Q}
$$

which makes $\hat{h}_{Q}$ normalized to unity: $\hat{h}_{Q}=1+O(q, t)$.

Let us give a list of explicit examples of these coefficients $\hat{h}_{Q}$, which illustrates their properties. In the tables below, included are only the diagrams for which the coefficients are non-vanishing. In the case of $r=1$ :

\footnotetext{
${ }^{1}$ In this letter we use the notation with "asymmetric" quantum numbers, $[x]_{t}=\frac{1-t^{x}}{1-t}$, which looks most adequate for torus knots. This is different from [1], where the symmetric choice was made, with $[x]_{t}^{[1]}=\frac{t^{x}-t^{-x}}{t-t^{-1}}$, differing in particular by the change $t \rightarrow t^{2}, q \rightarrow q^{2}$. The symmetric choice is good, it eliminates artificial square roots from formulas for generic knots. However, because of an additional $Z_{2}$-symmetry in the torus case, the asymmetric notation provides simplifications, and we use it in this short letter to make formulas as simple as possible.
} 
$\underline{(3,3 k+1):}$

\begin{tabular}{c|l} 
diagram $Q$ & coefficient $\hat{h}_{Q}$ \\
\hline$[3]$ & 1
\end{tabular}

$\underline{(4,4 k+1):}$

\begin{tabular}{c|l} 
diagram $Q$ & coefficient $\hat{h}_{Q}$ \\
\hline$[4]$ & 1
\end{tabular}

$\underline{(5,5 k+1):}$

\begin{tabular}{c|l} 
diagram $Q$ & coefficient $\hat{h}_{Q}$ \\
\hline$[5]$ & 1
\end{tabular}

and so on, i.e.

$$
\hat{h}_{Q}^{(m, 1)}= \begin{cases}1 & l(Q)=1 \\ 0 & \text { otherwise }\end{cases}
$$

The simplicity of this formula is stunning: it captures all the information about the superpolynomials of $(m, m k+1)$ knots in a single unity. As we will see below, from the point of view of the Hall-Littlewood reexpansion, $(m, m k+1)$-series is by no means distinguished: a similar phenomenon happens for the higher series, e.g. for $(m, m k+2),(m, m k+3)$ etc.

$\underline{(3,3 k+2):}$

\begin{tabular}{c|l} 
diagram $Q$ & coefficient $\hat{h}_{Q}$ \\
\hline$[3]$ & 1 \\
{$[2,1]$} & 1
\end{tabular}

$\underline{(5,5 k+2):}$

\begin{tabular}{c|l} 
diagram $Q$ & coefficient $\hat{h}_{Q}$ \\
\hline$[5]$ & 1 \\
{$[4,1]$} & 1 \\
{$[3,2]$} & 1
\end{tabular}

$\underline{(7,7 k+2):}$

\begin{tabular}{c|l} 
diagram $Q$ & coefficient $\hat{h}_{Q}$ \\
\hline$[7]$ & 1 \\
{$[6,1]$} & 1 \\
$5,2]$ & 1 \\
{$[4,3]$} & 1
\end{tabular}

and so on, i.e.

$$
\hat{h}_{Q}^{(m, 2)}= \begin{cases}1 & l(Q)=1 \\ 1 & l(Q)=2 \\ 0 & \text { otherwise }\end{cases}
$$

Remarkably, in these terms the case $m k+2$ looks just as simple as $m k+1$. All complications arise when one performs the linear transformation from $h_{Q}^{(m . r)}$ to $c_{Q}^{(m, r)}$. In [1] it was suggested to split $c_{Q}^{(m, r)}$ into the Adams coefficients and additional $\gamma$-factors, which were trivial for the HOMFLY polynomials, i.e. for $q=t$ : $c_{Q}^{(m, r)}=C_{Q}^{(m, r)} \gamma_{Q}^{(m, r)}$. Then for $r=1$ all the $\gamma^{\prime}$ 's are simple polynomials, $\gamma_{Q}^{(m, 1)} \sim \sum_{(i, j) \in Q} t^{i} q^{-j}$ (we use 
here the elegant reformulation of this result from [1] suggested in [13]). For $r=2$ these $\gamma$-factors are rather complicated rational functions, for example,

$$
\gamma_{[4,3]}^{(7,2)}=q^{4} \frac{q^{7} t^{3}+t^{2} q^{6}-t^{3} q^{5}+t q^{5}-q^{4} t^{3}+q^{4}+2 q^{4} t-q^{4} t^{2}-2 q^{3} t^{2}+q^{3}+q^{3} t-q^{2} t^{2}-q^{2} t+q^{2}-q t-1}{q^{2}-t}
$$

while the corresponding Adams coefficient is

$$
C_{[4,3]}^{(7,2)}=\frac{(1-t)(q-t)\left(q^{2}-t\right)}{\left(1-q^{2} t\right)\left(1-q^{3} t\right)\left(1-q^{4} t\right)}
$$

But this seeming complexity is actually nothing, but the result of the linear transformation from the HallLittlewood to MacDonald polynomials, and is a direct consequence of formulas (6).

Similarly, for $r=3$,

$(4,4 k+3):$

\begin{tabular}{c|l} 
diagram $Q$ & coefficient $\hat{h}_{Q}$ \\
\hline$[4]$ & 1 \\
{$[3,1]$} & $1+q$ \\
{$[2,2]$} & $1+t$ \\
{$[2,1,1]$} & $1+t$
\end{tabular}

$\underline{(5,5 k+3):}$

\begin{tabular}{c|l} 
diagram $Q$ & coefficient $\hat{h}_{Q}$ \\
\hline$[5]$ & 1 \\
{$[4,1]$} & $1+q$ \\
{$[3,2]$} & $1+q$ \\
{$[3,1,1]$} & $1+t$ \\
{$[2,2,1]$} & $1+t$
\end{tabular}

$(7,7 k+3):$

\begin{tabular}{c|l} 
diagram $Q$ & coefficient $\hat{h}_{Q}$ \\
\hline$[7]$ & 1 \\
{$[6,1]$} & $1+q$ \\
{$[5,2]$} & $1+q+q^{2}-q t$ \\
{$[4,3]$} & $1+q$ \\
{$[5,1,1]$} & $1+t$ \\
$4,2,1]$ & $1+q$ \\
$3,3,1]$ & $1+t$ \\
{$[3,2,2]$} & $1+t$
\end{tabular}

and so on, i.e.

$$
\hat{h}_{Q}^{(m, 3)}=\left\{\begin{array}{ll|}
1 & l(Q)=1 \\
1+t+(q-t)[\alpha]_{q} & l(Q)=2,3 \\
0 & \text { otherwise }
\end{array}\right.
$$

with $\alpha=\min \left(Q_{1}-Q_{2}, Q_{2}-Q_{3}\right)$. These examples clearly show the important role of the Hall-Littlewood basis.

\section{$3 \quad$ Generating functions}

As it often happens, the most adequate description of combinatorial information is given by generating functions. In our case, the combinatorial objects under consideration are the coefficients $\hat{h}_{Q}$. It is, therefore, convenient to pass from explicit formulas for $\hat{h}_{Q}$, which depend on integer variables $Q_{1}, Q_{2}, \ldots$, to generating functions which depend on continuous variables $x_{1}, x_{2}, \ldots$ This is achieved by summing over all diagrams $Q$ with appropriate weights $\omega_{Q}$ :

$$
\check{\rho}_{m}\left(x_{1}, \ldots, x_{r}\right)=\sum_{|Q|=m} \omega_{Q} \hat{h}_{Q} x_{1}^{Q_{1}} \ldots x_{r}^{Q_{r}}
$$

There is of course some freedom in the choice of the weights $\omega_{Q}$ : it can be used to simplify the generating functions as much as possible. In our case, the weight that gives the simplest answer comes from the HallLittlewood theory, it is essentially the inverse quadratic norm of the Hall-Littlewood polynomials:

$$
\omega_{Q}=\prod_{j} \frac{1}{\left[m_{j}(Q)\right]_{t} !}=(1-t)^{l(Y)}\left\|L_{Q}\right\|^{2}
$$


where $m_{j}(Y)=$ the number of rows with length $j$ in the diagram $Y$, and the quantum factorial is defined as $[x]_{t} !=[1]_{t} \ldots[x]_{t}$. This is the norm which also appears in the Cauchy formula

$$
\exp \left(\sum_{k} \frac{z^{k}}{k}\left(1-t^{k}\right) p_{k} \bar{p}_{k}\right)=\sum_{R} \frac{z^{|R|}}{\left\|L_{Q}\right\|^{2}} L_{R}\{p\} L_{R}\{\bar{p}\}
$$

It is further convenient to sum over all indices $m$ coprime with $r$ :

$$
\rho\left(x_{1}, \ldots, x_{r} \mid z\right)=\sum_{m \perp r} z^{m} \check{\rho}_{m}\left(x_{1}, \ldots, x_{r}\right)
$$

With these conventions, the 1-point function becomes

$$
\rho(x \mid z)=\frac{x z}{1-x z}
$$

the 2-point function becomes

$$
\rho\left(x_{1}, x_{2} \mid z\right)=\frac{x_{1} z}{\left(1-x_{1}^{2} z^{2}\right)\left(1-x_{1} x_{2} z^{2}\right)}
$$

the 3-point function becomes

$$
\rho\left(x_{1}, x_{2}, x_{3} \mid z\right)=\frac{z x_{1}\left(1-q t x_{1}^{4} x_{2}^{2} z^{6}\right)\left(1+z\left(x_{1}+x_{2}\right)\left(1+x_{1} x_{2} z^{2}\right)+x_{1}^{2} x_{2}^{2} z^{4}\right)}{\left(1-x_{1}^{3} z^{3}\right)\left(1-q x_{1}^{2} x_{2} z^{3}\right)\left(1-x_{1}^{3} x_{2}^{3} z^{6}\right)\left(1-x_{1} x_{2} x_{3} z^{3}\right)}
$$

In general, $\rho$ is a polynomial of degree $(r-1)(r-2) / 2$ in $t$ which provides a kind of "separation of variables" $q$ and $t$. In particular, the 4-point function consists of four different terms. The dependence on $z$ can be easily restored by dimensional argument, hence, we omit it from the formulas below.

$$
\rho\left(x_{1}, x_{2}, x_{3}, x_{4}\right)=\frac{\left(x_{1}+x_{1} x_{2} x_{3}\right)\left(N_{0}+N_{1} t+N_{2} t^{2}+N_{3} t^{3}\right)}{D\left(x_{1}, x_{2}, x_{3}, x_{4}\right)}
$$

where

$$
\begin{aligned}
& D\left(x_{1}, x_{2}, x_{3}, x_{4}\right)=\left(1-x_{1}^{2}\right)\left(1-q x_{1} x_{2}\right)\left(1-x_{1} x_{2}\right)\left(1-q^{2} x_{1}^{3} x_{2}\right) \times \\
& \times\left(1-x_{1}^{2} x_{2}^{2} x_{3}^{2}\right)\left(1-q^{2} x_{1}^{2} x_{2} x_{3}\right)\left(1-q x_{1}^{2} x_{2} x_{3}\right)\left(1-q^{2} x_{1}^{3} x_{2}^{3} x_{3}^{2}\right)\left(1-x_{1} x_{2} x_{3} x_{4}\right) \\
& N_{0}=\left(1-q^{2} x_{1}^{3} x_{2}^{2} x_{3}\right)\left(1-q^{2} x_{1}^{5} x_{2}^{3} x_{3}^{2}\right) \\
& N_{1}=q x_{1} x_{2}-q x_{1}^{3} x_{2}-q^{2} x_{1}^{3} x_{2}^{2} x_{3}-q^{3} x_{1}^{4} x_{2}^{2}-q x_{1}^{3} x_{2}^{3} x_{3}^{2}+q^{2} x_{1}^{4} x_{2}^{3} x_{3}+q^{3} x_{1}^{5} x_{2}^{3}-q^{3} x_{1}^{4} x_{2}^{4} x_{3}^{2}+q^{4} x_{1}^{5} x_{2}^{4} x_{3}+ \\
& +q^{3} x_{1}^{5} x_{2}^{5} x_{3}^{2}+q^{3} x_{1}^{6} x_{2}^{3} x_{3}^{3}+q^{3} x_{1}^{6} x_{2}^{4} x_{3}^{2}-q^{4} x_{1}^{7} x_{2}^{4} x_{3}+q^{5} x_{1}^{7} x_{2}^{4} x_{3}^{3}-q^{4} x_{1}^{8} x_{2}^{4} x_{3}^{2}-q^{4} x_{1}^{7} x_{2}^{6} x_{3}^{3}-q^{3} x_{1}^{8} x_{2}^{5} x_{3}^{3}- \\
& -q^{5} x_{1}^{8} x_{2}^{6} x_{3}^{2}-q^{5} x_{1}^{9} x_{2}^{4} x_{3}^{3}-q^{4} x_{1}^{8} x_{2}^{6} x_{3}^{4}+q^{5} x_{1}^{9} x_{2}^{5} x_{3}^{4}-q^{5} x_{1}^{9} x_{2}^{6} x_{3}^{5}+q^{6} x_{1}^{11} x_{2}^{7} x_{3}^{4}+ \\
& +[2]_{q} q^{2} x_{1}^{4} x_{2}^{3} x_{3}\left(-q^{4} x_{1}^{5} x_{2}^{3} x_{3}^{2}\left(-1+x_{2} x_{1}^{3} x_{3}^{2}\right)+q^{2} x_{1}^{3} x_{2}\left(1+x_{2}^{2} x_{3}^{2}\right)\left(1+x_{1} x_{3}\right)+q x_{1}^{3} x_{2} x_{3}^{2}-\left(1+x_{1} x_{3}\right)\right)- \\
& -[3]_{q} q x_{1}^{4} x_{2}^{2} x_{3}^{2} \\
& N_{2}=q^{2} x_{1}^{3} x_{2}^{2} x_{3}-q^{3} x_{1}^{5} x_{2}^{3}+q^{3} x_{1}^{5} x_{2}^{4} x_{3}-q^{4} x_{1}^{6} x_{2}^{3} x_{3}-q^{3} x_{1}^{5} x_{2}^{5} x_{3}^{2}-q^{3} x_{1}^{6} x_{2}^{3} x_{3}^{3}-q^{5} x_{1}^{6} x_{2}^{4} x_{3}^{2}-q^{4} x_{1}^{7} x_{2}^{3} x_{3}^{2}-q^{4} x_{1}^{6} x_{2}^{5} x_{3}^{3}+ \\
& +q^{3} x_{1}^{7} x_{2}^{5} x_{3}^{2}-q^{4} x_{1}^{7} x_{2}^{5} x_{3}^{4}+q^{5} x_{1}^{8} x_{2}^{5} x_{3}^{3}+q^{5} x_{1}^{8} x_{2}^{6} x_{3}^{2}+q^{5} x_{1}^{9} x_{2}^{4} x_{3}^{3}+q^{4} x_{1}^{9} x_{2}^{5} x_{3}^{4}-q^{5} x_{1}^{10} x_{2}^{5} x_{3}^{3}+q^{5} x_{1}^{9} x_{2}^{6} x_{3}^{5}+ \\
& +q^{6} x_{1}^{10} x_{2}^{6} x_{3}^{4}-q^{7} x_{1}^{11} x_{2}^{6} x_{3}^{3}-q^{5} x_{1}^{10} x_{2}^{7} x_{3}^{5}-q^{6} x_{1}^{11} x_{2}^{7} x_{3}^{4}-q^{7} x_{1}^{11} x_{2}^{8} x_{3}^{5}+q^{7} x_{1}^{13} x_{2}^{8} x_{3}^{5}+ \\
& +[2]_{q} q x_{1}^{2} x_{2}^{2}\left(\left(-1+x_{2} x_{1}^{3} x_{3}^{2}\right)+q^{2} x_{1}^{4} x_{3} x_{2}^{2}\left(1+x_{2}^{2} x_{3}^{2}\right)\left(1+x_{1} x_{3}\right)+q^{3} x_{2}^{2} x_{3}^{2} x_{1}^{5}-q^{4} x_{1}^{7} x_{2}^{4} x_{3}^{3}\left(1+x_{1} x_{3}\right)\right)- \\
& -[3]_{q} q^{5} x_{1}^{10} x_{2}^{7} x_{3}^{3} \\
& N_{3}=-q^{4} x_{1}^{6} x_{2}^{4} x_{3}^{2}\left(1-q^{2} x_{1}^{3} x_{2}^{2} x_{3}\right)\left(1-q^{2} x_{1}^{5} x_{2}^{3} x_{3}^{2}\right)
\end{aligned}
$$




\section{Conclusion}

We described what we believe is an important parametrization of the coefficients $c_{Q}^{(m, r)}$ in the MacDonald expansion (1) of the extended superpolynomials of ref.[1] for the torus knots.

The following table shows the structure of our calculation:

$$
\begin{gathered}
P_{m, m k+r}(A)=\sum_{Q \vdash m} c_{Q}^{(m, r)} q^{-k \nu\left(Q^{T}\right)} t^{k \nu(Q)} M_{Q}^{*}(A) \\
\downarrow \\
\mathcal{P}_{m, m k+r}\{p\}=\sum_{Q \vdash m} c_{Q}^{(m, r)} q^{-k \nu\left(Q^{T}\right)} t^{k \nu(Q)} M_{Q}\{p\}=e^{k \hat{W}} \mathcal{P}_{m, r}\{p\} \\
\downarrow \\
\mathcal{P}_{m, r}(p)=\sum_{Q \vdash m} c_{Q}^{(m, r)} M_{Q}\{p\}=\sum_{\substack{Q \vdash m \\
l(Q) \leq r}} h_{Q}^{(m, r)} L_{Q}\{p\} \\
\downarrow P_{m, r}(A)=\sum_{\substack{Q \vdash m \\
l(Q) \leq r}} h_{Q}^{(m, r)} L_{Q}^{*}(A)=A^{r-m} P_{r, m}(A)=\sum_{Y \vdash r} c_{Y}^{\left(r, r^{\prime}\right)} q^{-k^{\prime} \nu\left(Y^{T}\right)} t^{k \nu(Y)} A^{r-m} M_{Y}^{*}(A)
\end{gathered}
$$

The ordinary torus superpolynomial (18) is expanded, as a function of $A$, into the MacDonald dimensions $M_{Q}^{*}(A)$. For $m \geq 4$ this expansion becomes ambiguous (the ambiguity is absent for the torus HOMFLY polynomials, where only the hook diagrams $Q=[m-i, \underbrace{1, \ldots, 1}_{i}]$ contribute). The extended superpolynomial (19) is no longer ambiguous, but instead it is not an invariant, it depends on the choice of the braid representation. Still, we believe that $i t$ is the central object to study in the refined Chern-Simons theory $[15,11]$. For the torus knots, the extended superpolynomial (19) comes directly in the $W$-representation [5], which explicitly describes "evolution" in the $k$-parameter, so that the only unknown piece of the answer is the "initial" condition at $k=0$, i.e. the extended superpolynomial (20) of the knot $T[m, r]$ with $r<m$. If reduced back to the ordinary superpolynomials, $P_{m, r}(A)$ is related by the obvious duality $T[m, n]=T[n, m]$ to another superpolynomial, $P_{r, m}(A)$, with less strands $r<m$ than the original one. Accordingly, the sum over $Q$ for $\mathcal{P}_{m, r}\{p\}$ is actually "smaller" than for $k \neq 0$. As an explicit manifestation of this fact, there is a special basis, where the only contributing $Q$, while still having the size $m$, have no more than $r$ rows, $l(Q) \leq r$. It turns out that this special basis is formed not by MacDonald, but by the simpler. Hall-Littlewood polynomials $L_{Q}\{p\}$ (the same property persists in the basis of "rescaled" Schur functions, but the expansion coefficients are still more sophisticated in this case).

Note that we have discussed so far only the Hall-Littlewood decomposition of the $(m, r)$ superpolynomials with $r<m, m \perp r$ and the "initial" condition given at $k=0$. However, as we already mentioned there is a duality symmetry $T[m,-n] \leftrightarrow T[m, n]$, i.e. $T[m, r] \leftrightarrow T[m, m-r]$ and, simultaneously, $q \leftrightarrow q^{-1}, t \leftrightarrow t^{-1}$. It allows one to describe also the case $k=-1$. Namely, in addition to (4), one now has

$$
\mathcal{P}_{m, r-m}\{p\}=\sum_{Q \vdash m} c_{Q}^{(m, r)} q^{k \nu\left(Q^{T}\right)} t^{-k \nu(Q)} M_{Q}\{p\}=\sum_{\substack{Q \vdash m \\ l(Q) \leq r}} \widetilde{h}_{Q}^{(m, r)} \widetilde{L}_{Q}\{p\}
$$

where $\widetilde{L}_{Q}$ and $\widetilde{h}_{Q}$ are the dual Hall-Littlewood polynomials and coefficients, respectively:

$$
\widetilde{L}_{Q}\{p\}=\left.L_{Q}\{p\}\right|_{t=t^{-1}}, \quad \quad \widetilde{h}_{Q}^{(m, r)}=\left.q^{m(m-1) / 2} \cdot h_{Q}^{(m, m-r)}\right|_{\substack{t=t^{-1} \\ q=q^{-1}}}
$$

Further comments, explanations and examples (which substantially extend the original list in [1]) will be presented elsewhere. We believe that appearance of two different expansion bases: one for the $n$-evolution, 
another one for its "initial" condition (i.e. for the $m$-evolution) is not an accident, and reflects some additional duality structure. Even more interesting is that this second evolution involves only the Hall-Littlewood expansion, what implies a possible existence of still another deformation of the entire construction, perhaps, going as far as the Kerov-Askey-Wilson character expansion. The situation here looks reminiscent of the one with double elliptic deformations of integrable systems [14].

Extension of these results to other representations (colored superpolynomials) looks straightforward for the torus knots, but remains to be done. Extension to similar series of non-torus knots (a la [7]) is a far more interesting, though a little less trivial exercise. Last but not least, a hidden algebraic (integrable) structure behind the extended superpolynomials (again, al la [7]) is to be revealed.

\section{Acknowledgements}

We are indebted to Ivan Cherednik, Petr Dunin-Barkovsky, Eugene Gorsky, Sergei Gukov and Alexei Oblomkov for very useful and inspiring communications. They also kindly compared some implications of our general formulas with their own answers, obtained by different methods. We understand that Alexey Oblomkov recently found an alternative effective description of all the coefficients $c_{Q}^{(m, r)}$.

Our work is partly supported by Ministry of Education and Science of the Russian Federation under contract 14.740.11.0608, by the Dynasty Foundation, by RFBR grant 10-01-00536 and by joint grants 11-02-90453-Ukr, 09-02-93105-CNRSL, 12-02-91000-ANF, 12-02-92108-Yaf-a, 11-01-92612-Royal Society. The work of Sh.Shakirov is supported in part by Berkeley Center for Theoretical Physics, by the National Science Foundation (award number 0855653), by the Institute for the Physics and Mathematics of the Universe, by the US Department of Energy under Contract DE-AC02-05CH11231. 


\section{References}

[1] P.Dunin-Barkowski, A.Mironov, A.Morozov, A.Sleptsov and A.Smirnov, arXiv:1106.4305

[2] M.Khovanov and L.Rozhansky, Fund. Math. 199 (2008) 1, math.QA/0401268; Geom. Topol. 12 (2008) 1387, math.QA/0505056

[3] S.Gukov, A.Schwarz and C.Vafa, Lett.Math.Phys. 74 (2005) 53-74, hep-th/0412243

N.M.Dunfield, S.Gukov and J.Rasmussen, Experimental Math. 15 (2006) 129-159, math/0505662

S.Gukov and J.Walcher, hep-th/0512298

S.Gukov, A.Iqbal, C.Kozcaz and C.Vafa, arXiv:0705.1368

S.Gukov, arXiv:0706.2369

N.Carqueville and D.Murfet, arXiv:1108.1081

S.Gukov and M.Stosic, arXiv:1112.0030

[4] A.Mironov, A.Morozov and S.Natanzon, Theor.Math.Phys. 166 (2011) 1-22, arXiv:0904.4227; Journal of Geometry and Physics, 62 (2012) 148-155 arXiv:1012.0433

[5] A.Morozov and Sh.Shakirov, JHEP 0904 (2009) 064, arXiv:0902.2627; Mod.Phys.Lett. A24 (2009) 26592666, arXiv:0906.2573;

G.Borot, B.Eynard, M.Mulase and B.Safnuk, arXiv:0906.1206

A.Alexandrov, arXiv:1005.5715, arXiv:1009.4887

[6] M.Rosso and V.F.R.Jones, J. Knot Theory Ramifications, 2 (1993) 97-112

J.M.F.Labastida and M.Marino,J.Knot Theory Ramifications, 11 (2002) 173

X.-S.Lin and H.Zheng, Trans. Amer. Math. Soc. 362 (2010) 1-18, math/0601267

A.Brini, B.Eynard and M.Mariño, arXiv:1105.2012

[7] A.Mironov, A.Morozov and And.Morozov, arXiv:1112.2654; arXiv:1112.5754

[8] A.Morozov, arXiv:1201.4595

[9] E.Gorsky, arXiv:1003.0916

[10] A.Oblomkov, J.Rasmussen and V.Shende, arXiv:1201.2115 (with an Appendix by Eugene Gorsky)

[11] M.Aganagic and Sh.Shakirov, arXiv: 1105.5117

[12] I.Cherednik, arXiv:1111.6195

[13] Sh.Shakirov, arXiv:1111.7035

[14] H.Braden, A.Marshakov, A.Mironov and A.Morozov, Nucl.Phys., B573 (2000) 553, hepth/9906240 see also a brief review in: A.Mironov, Theor.Math.Phys., 129 (2001) 1581-1585, hep-th/0104253 A.Mironov and A.Morozov, Phys.Lett., B475 (2000) 71-76, hepth/9912088; hepth/0001168 H.Braden, A.Gorsky, A.Odesskii and V.Rubtsov, hep-th/0111066

[15] A.Iqbal, C.Kozcaz and C.Vafa, JHEP 0910 (2009) 069, hep-th/0701156 H.Awata and H.Kanno, Int.J.Mod.Phys. A24 (2009) 2253-2306, arXiv:0805.0191

\footnotetext{
${ }^{2} \mathrm{~A}$ remarkably simple suggestion for the $\gamma$-factors in this paper does not seem literally applicable to the torus knots, it does not even reproduce the HOMFLY polynomials. It is so attractive, however, and it is non-trivial that such a simple ansatz provides polynomials with positive integer coefficients. Therefore, there should be a prominent place for it in the future theory of superpolynomials.
} 